\title{
White LED light production by using dibromoperylene derivatives in down conversion of energy
}

\author{
Erkan AKSOY ${ }^{1}$, Nuriye DEMIR ${ }^{1}$, Canan VARLIKLI ${ }^{2 *}$ \\ ${ }^{1}$ Ege University, Solar Energy Institute, 35100, Bornova, Izmir, Turkey \\ ${ }^{2}$ İzmir Institute of Technology, Department of Photonics, 35430, Urla, Izmir, Turkey \\ *Corresponding Author. Tel: +90 232 7507732; cananvarlikli@iyte.edu.tr
}

\begin{abstract}
Perylene derivatives of N,N'-bis(2,6-diisopropylphenyl)-1,6(7)-dibromoperylene-3,4,9,10tetracarboxylic diimide (DBrPDI) and 1,7-dibromo perylene-3,4,9,10-tetracarboxylic acid butyl ester (DBrPTE) were synthesized, their structural and optical characterizations were performed. Both DBrPDI and DBrPTE, which were utilized as wavelength converters on a GaN/InGaN based blue LED, were singlet sensitizers and had molar absorptivity constants around $10^{4} \mathrm{Lmol}^{-1} \cdot \mathrm{cm}^{-1}$. The blue LED that was used had Commission internationale de l'eclairage (CIE) coordinates of $\mathrm{x}, \mathrm{y}=0.13,0.06$, power and luminous efficiencies of $2.7 \mathrm{~lm} / \mathrm{W}$ and $6.6 \mathrm{~cd} / \mathrm{A} @ 5 \mathrm{~mA}$, respectively. Since the fluorescence quantum yield of DBrPDI was much higher than that of DBrPTE, a meaningful white light efficiency was obtained with the diimide derivative. The use of DBrPDI resulted in white light with CIE coordinates of $\mathrm{x}, \mathrm{y}=0.34,0.29$ and luminous efficiency of $6.35 \mathrm{~cd} / \mathrm{A} @ 5 \mathrm{~mA}$, respectively. Using DBrPDI in a resin matrix resulted in more than 3 orders of magnitude increase in the luminous efficiency value $(23.0 \mathrm{~cd} / \mathrm{A} @ 5 \mathrm{~mA})$ while the CIE coordinates remained the same. Whenever the applied current reached $20 \mathrm{~mA}$, a CRI value of $\mathrm{Ra}=90$ was obtained.
\end{abstract}

Keywords: Organic phosphor; Perylene; PMMA; Downconversion; White light 


\section{Introduction}

Light emitting diodes (LEDs) are known to have great potential as energy efficient lighting systems. Although electroluminescence (EL) abilities of inorganic semiconductors and devices similar to LEDs were known in 1950s, their potential in general lighting application was realized in 1990s when S. Nakamura developed the first nitride-based blue emitting LED [1,2]. Optimized combination of orange-red phosphor and blue emitting LED provides white light generation [3]. Although Cerium dopped Yttrium Aluminium Garnet (Ce:YAG) has many disadvantages [e.g. poor color rendering indices (CRI) of 75, high correlated color temperature (CCT) of $7756 \mathrm{~K}$ and cool white light with corresponding to Commission internationale de l'eclairage (CIE) chromaticity coordinates of $(0.292,0.325)$ ] $[4,5]$, it represents the most popular phosphor material used by the corresponding industry mostly because of the comfort of its well-known properties. Many research groups are working on developing alternative phosphor materials which would provide better CRI and CCT values and warm white light [5-8]. However, as Europium is used in most of them and because of the cost of this element, application of these efficient phosphors to industry remains limited.

Recently, not only because of their economic impacts but also because of their environmentally friendly natures, organic dyes started to gain attention [9-14]. Great photo and thermal stabilities, high molar absorptivity constants $(\varepsilon)$ in the visible region and high fluorescence quantum yields $\left(\Phi_{\mathrm{PL}}\right)$ of $3,4,9$, 10-perylenetetracarboxylic-diimide (PDI) derivatives [15-17] make them one of the best organic dye alternatives for down conversion of energy produced by the blue LED. However, the $\Phi_{\mathrm{PL}}$ value of PDIs decreases in film phase due to the $\pi-\pi$ aggregation originating from the ring plane [18]. To overcome this problem, two main approaches can be followed; i) determining the optimum concentration of the dye to obtain maximum photoluminescence (high dye concentrations may induce self-quenching mechanism that maybe followed by aggregation induced quenched photoluminescence [19]) and ii) changing the intermolecular distance between the ring planes by the use of dopants, incorporation of the dye in a host matrix or deterioration of ring plane by bulky substituents. In order to interrupt $\pi-\pi$ stacking between the perylene rings, second approach was followed in this study, i.e. functionalization of the perylene ring from its 1, 6 and/or 7 positions [20] and incorporation of the dye in polymethyl methacrylate (PMMA).

Two perylene derivatives of N,N'-bis(2,6-diisopropylphenyl)-1,6(7)-dibromoperylene3,4,9,10-tetracarboxylic diimide (DBrPDI) and 1,7-dibromo perylene-3,4,9,10tetracarboxylic acid butyl ester (DBrPTE) were synthesized and used as wavelength 
converters on a Gallium Nitride/Indium Gallium Nitride ( $\mathrm{GaN} / \mathrm{InGaN})$ based blue LED. DBrPDI presented better performance because of its higher photoluminescence quantum yield. Whenever this derivative was incorporated in PMMA matrix and applied on the blue LED, white light with CIE coordinates of $\mathrm{x}, \mathrm{y}=0.34,0.29$ and CRI of 90 was obtained.

\section{Experimental}

\section{Materials and Instruments}

2,6-diisopropylaniline, potassium carbonate, 1-iodobutane and 1,8-Diazabicyclo(5.4.0)undec7-ene (DBU) were from Acros Organics, VWR Chemicals, ABCR Chemicals and TCI Chemicals, respectively. Ethanol absolute, hydrochloric acid, bromine, iodine, 1-butanol, methanol, acetonitrile (ACN), hexane, chloroform, dichlorometane (DCM), N,N-dimethylformamide (DMF), acetic acid, 1-methyl-2-pyrrolidinone, silica gel 0.040-0.063 mm were obtained from Sigma-Aldrich and perylene-3,4,9,10-tetracarboxylic dianhydride (PTCDA) was purchased from Fluka. The blue LED based on $\mathrm{GaN} / \mathrm{InGaN}$ and polymethyl methacrylate (PMMA) were purchased from PROLED and Ossila, respectively.

${ }^{1} \mathrm{H}-\mathrm{NMR}$ spectra were recorded on a Bruker $400 \mathrm{MHz}$ spectrometer. Infrared (FTIR) spectra were obtained by using a PerkinElmer Spectrum BX-FTIR spectrophotometer. UV-Vis absorption spectra were recorded by using Analytik Jena S $600 \mathrm{UV}-\mathrm{Vis}$ spectrophotometer. Photoluminescence $(\mathrm{PL})$, absolute photoluminescence quantum yield $\left(\Phi_{\mathrm{PL}}\right)$ and life time $\left(\tau_{\mathrm{f}}\right)$ measurements were performed by using Edinburgh Instruments FLS920P spectrophotometer. EL characteristics of the devices were measured by Keithley 2400 source meter and Hamamatsu C9920-12 external quantum efficiency measurement system utilizing an integrating sphere.

\section{Synthesis of perylene derivatives}

Perylene derivatives of 1,7-Dibromoperylene-3,4,9,10-tetracarboxytetrabutylester (DBrPTE) and $\quad \mathrm{N}, \mathrm{N}^{\prime}$-Bis(2,6-diisopropylphenyl)-dibromoperylene-3,4,9,10-tetracarboxybisimide (DBrPDI) were synthesized by following the procedure in literature [21]. The synthetic pathway is summarized in Scheme 1 and below: 


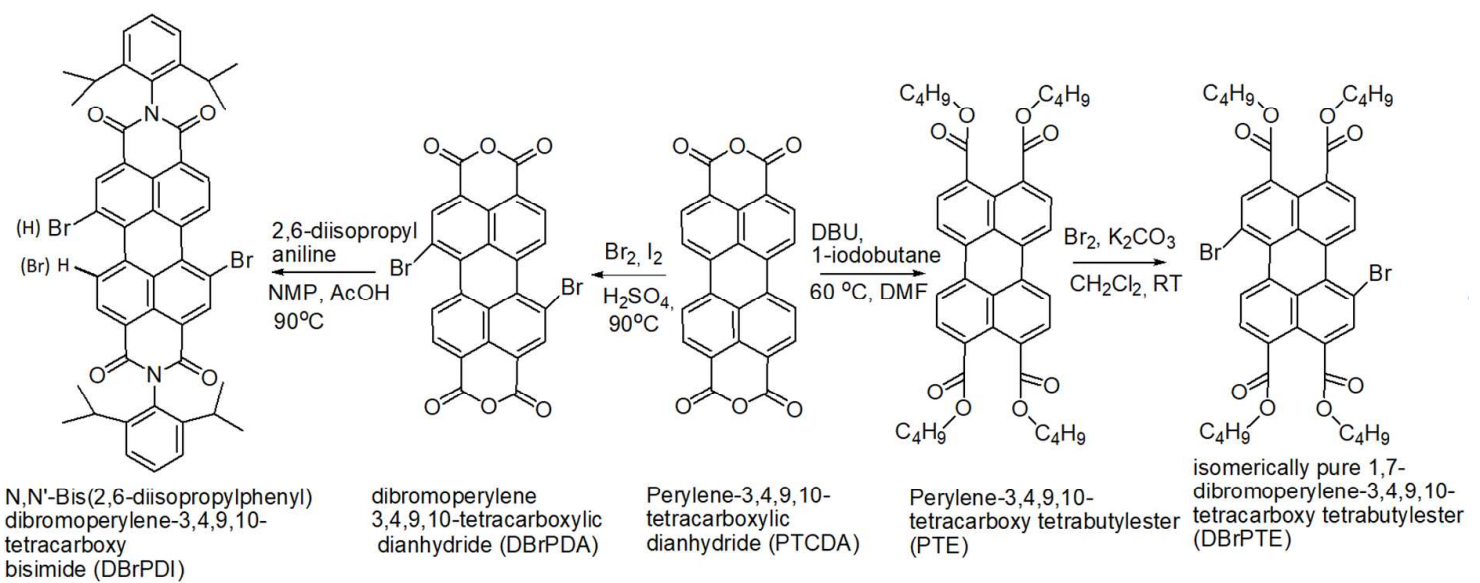

Scheme 1 Synthetic route of brominated perylene derivatives.

Synthesis of perylene-3,4,9,10-tetracarboxytetrabutylester (PTE): $1 \mathrm{~g}$ (2.54 mmol) of perylene-3,4,9,10-tetracarboxylic dianhydride was placed in a $50 \mathrm{~mL}$ two-neck round bottom flask, DBU $(1.52 \mathrm{~mL})$, n-butanol $(1.86 \mathrm{~mL})$ and DMF $(13 \mathrm{~mL})$ were added to the reaction flask and they were stirred in Ar atmosphere at $60{ }^{\circ} \mathrm{C}$ for $30 \mathrm{~min}$. Then 1-iodobutane $(1.84 \mathrm{~g}$, $10 \mathrm{mmol})$ mixed with DMF $(10 \mathrm{~mL})$ was added. The mixture was stirred at $60{ }^{\circ} \mathrm{C}$ for a further 3 hours and then cooled to room temperature. The reaction mixture was slowly added into distilled water $(100 \mathrm{~mL})$ and the solid was filtered. The solid material was dried $\left(80^{\circ} \mathrm{C}\right)$ and purified by column chromatography (silica gel, DCM). Yield: 1.52g (92\%). FTIR (KBr) (Figure 1): $U_{\max }=\left(\mathrm{C}_{\mathrm{Ar}}-\mathrm{H}\right): 2959 \mathrm{~cm}^{-1} ;(\mathrm{C}-\mathrm{H}): 2872 \mathrm{~cm}^{-1},(\mathrm{C}=\mathrm{O}): 1724 \mathrm{~cm}^{-1},(\mathrm{C}-\mathrm{O}): 1276-$ $1172 \mathrm{~cm}^{-1} .{ }^{1} \mathrm{H}$ NMR (400 MHz, Chloroform-d, TMS/ppm) $\delta: 7.92$ (d, J: $\left.2.0 \mathrm{~Hz}, 4 \mathrm{H}\right), 7.84$ (d, $J: 2.0 \mathrm{~Hz}, 4 \mathrm{H}), 4.36(\mathrm{t}, J: 2.0 \mathrm{~Hz}, 8 \mathrm{H}), 1.85-1.78$ (m, J: $2.0 \mathrm{~Hz}, 8 \mathrm{H}), 1.57-1.48$ (m, J: $2.0 \mathrm{~Hz}$, $8 \mathrm{H}), 1.02 \mathrm{ppm}(\mathrm{t}, J: 2.0 \mathrm{~Hz}, 12 \mathrm{H})$.

Synthesis of regioisomerically pure 1,7-dibromoperylene-3,4,9,10-tetracarboxytetrabutylester (DBrPTE): PTE (0.35 g, $0.53 \mathrm{mmol}), \mathrm{DCM}(5 \mathrm{~mL})$ and $\mathrm{K}_{2} \mathrm{CO}_{3}(0.75 \mathrm{~g}, 5.4 \mathrm{mmol})$ were added into a $10 \mathrm{~mL}$ round bottom flask. The reaction mixture was stirred at room temperature for 30 min, and then bromine $(0.35 \mathrm{~mL})$ was added slowly and stirred for 24 hours at room temperature. Excess bromine was removed by passing air through the reaction flask in the fume hood. The solid mixture was dissolved with DCM and ACN and then allowed to crystallize. Yield: 0.1g (25\%). FTIR (KBr) (Figure 1): $\mathrm{U}_{\max }=\left(\mathrm{C}_{\mathrm{Ar}}-\mathrm{H}\right): 2956 \mathrm{~cm}^{-1} ;(\mathrm{C}-\mathrm{H})$ : $2869 \mathrm{~cm}^{-1},(\mathrm{C}=\mathrm{O}): 1717 \mathrm{~cm}^{-1},(\mathrm{C}-\mathrm{O}): 1300-1175 \mathrm{~cm}^{-1} .{ }^{1} \mathrm{H}$ NMR (400 MHz, Chloroform-d, TMS/ppm) (Figure 2) $\delta: 8.88(\mathrm{~d}, J: 2.0 \mathrm{~Hz}, 4 \mathrm{H}), 8.27$ (s, 4H), 8.36 (d, J: $2.0 \mathrm{~Hz}, 8 \mathrm{H}), 4.35$ (t, $J: 2.0 \mathrm{~Hz}, 8 \mathrm{H}), 1.83-1.76(\mathrm{~m}, J: 2.0 \mathrm{~Hz}, 8 \mathrm{H}), 1.55-1.46$ (t, $J: 2.0 \mathrm{~Hz}, 8 \mathrm{H}), 1.00$ ppm (t, J: 2.0 $\mathrm{Hz}, 12 \mathrm{H})$.

Synthesis of N,N'-Bis(2,6-diisopropylphenyl)-dibromoperylene-3,4,9,10-tetracarboxybisimide 
(DBrPDI): PTCDA $(5 \mathrm{~g}, 12.7 \mathrm{mmol})$ was added to a $250 \mathrm{~mL}$ two-neck round bottom flask. Then, $\mathrm{H}_{2} \mathrm{SO}_{4}$ was added slowly and stirred at room temperature for 24 hours. After iodine $(0.2 \mathrm{~g}, 0.78 \mathrm{mmol})$ was added into the reaction flask, the reaction temperature was increased to $85^{\circ} \mathrm{C}$ and kept for an hour. Bromine $(3.9 \mathrm{~mL})$ addition was performed and allowed mixing for 15 hours. The reaction mixture was cooled to room temperature and poured into pure water. The crude material was filtered, washed with distilled water and dried. The product was named as DBrPDA but could not be characterized by NMR because of its solubility problem and was used as crude product in the synthesis of DBrPDI. DBrPDA (0.46 g) was added into a reaction flask. Acetic acid $(0.3 \mathrm{~mL}), \mathrm{NMP}(10 \mathrm{~mL})$ and 2,6-diisopropylaniline $(1 \mathrm{~mL})$ was added to the flask in Ar atmosphere and the mixture was stirred under reflux for 4 days at 120 ${ }^{\circ} \mathrm{C}$. The mixture was cooled to room temperature and then poured into pure water. The crude material was filtered and washed with pure water. Purified by column chromatography (silica gel, DCM:Hexane 2:1. Note that the product was 1,6- and 1,7- dibromine mixture. Regioisomerically pure derivatives could not be obtained). Yield: 0.43g (60\%). FTIR (KBr) (Figure 1): $U_{\max }=\left(\mathrm{C}_{\mathrm{Ar}} \mathrm{H}\right): 2960,2928 \mathrm{~cm}^{-1} ;(\mathrm{C}-\mathrm{H}): 2866 \mathrm{~cm}^{-1},(\mathrm{C}=\mathrm{O}): 1709,1669 \mathrm{~cm}^{-1},\left(\mathrm{C}_{\mathrm{Ar}}{ }^{-}\right.$ $\left.\mathrm{C}_{\mathrm{Ar}}\right): 1588 \mathrm{~cm}^{-1},(\mathrm{HC}=\mathrm{C}): 1386,1333 \mathrm{~cm}^{-1},(\mathrm{C}-\mathrm{N}): 1242 \mathrm{~cm}^{-1} \cdot{ }^{1} \mathrm{H}$ NMR $(400 \mathrm{MHz}$, Chloroform-d, TMS/ppm) (Figure 3) $\delta$ 9.58-9.56 (d, J: 8 Hz, 2H), 9.03 (s, 2H), 8.82-8.80 (d, J: $8 \mathrm{~Hz}, 2 \mathrm{H}), 7.52$ (t, J: $8 \mathrm{~Hz}, 2 \mathrm{H}), 7.38$ (d, J: $8 \mathrm{~Hz}, 4 \mathrm{H}), 2.75$ (m, 4H), 1.20-1.19 ppm (d, 24H).

\section{Preparation of downconversion solution and experimental setup}

DBrPDI and DBrPTE were dissolved in chloroform with a concentration of $10 \mathrm{mg} / \mathrm{ml}$ and drop casted on GaN/InGaN based blue LED which had a peak emission wavelength of 465 $\mathrm{nm}$, CIE co-ordinates of $(0.13,0.06)$ and power and current efficiencies of $2.7 \mathrm{~lm} / \mathrm{W}$ and 6.6 cd/A@5mA, respectively. DBrPDI solution was further mixed with PMMA resin with a ratio of 1:2 and kept in ultrasonic bath for $30 \mathrm{~min}$. This homogeneous mixture was also drop casted on the blue LED. The blue LED used and prepared samples were named as InGaN and InGaN/DBrPTE, InGaN/DBrPDI, InGaN/DBrPDI:resin, respectively.

\section{Results and Discussion}

\section{Absorption and Photoluminescence Studies}

Absorption and PL spectra of synthesized perylene derivatives are provided in Figure 4 and their properties are summarized in Table. DBrPDI has a red shifted absorption compared to DBrPTE due to its approximately $6 \mathrm{kcal} / \mathrm{mol}$ lower singlet energy $\left(\mathrm{E}_{\mathrm{s}}\right)$ state. Both have absorption and PL peaks which are typical for the tetraester and diimide perylene derivatives; 
maximum absorption wavelengths $\left(\lambda_{\text {abs }}\right)$ correspond to the $\pi \rightarrow \pi^{*}$ and $n \rightarrow \pi^{*}$ transitions (Figure $4 \mathrm{a}$ ) and maximum emission wavelengths $\left(\lambda_{\mathrm{ems}}\right)$ are assigned to the $\mathrm{S}_{1} \rightarrow \mathrm{S}_{0}$ relaxation (Figure 4b) [22].

The absolute $\Phi_{\mathrm{PL}}$ values of both of the synthesized dyes are measured by using an integrating sphere attached to the spectrophotometer. The measured absolute values are lower than the relative $\Phi_{\mathrm{PL}}$ values reported in literature [21]. This situation was expected because following the exact conditions of the $\Phi_{\mathrm{PL}}$ reference (e.g. using the same excitation wavelength, making solvent or environment correction) occasionally escape from the attention of researchers while reporting the relative $\Phi_{\mathrm{PL}}$ values, and most commonly, relative $\Phi_{\mathrm{PL}}$ values reported in one study is accepted as the reference in calculation of other materials' relative $\Phi_{\mathrm{PL}}[21,22]$. The measured fluorescence life times $\left(\tau_{\mathrm{f}}\right)$ are at ns scale and the molar absorptivity constants (ع) are around $10^{4} \mathrm{M}^{-1} \mathrm{~cm}^{-1}$ for both of them. Although the absorption spectrum of DBrPTE exhibits a better overlap with a higher $\varepsilon$ value at the corresponding wavelength $\left(\lambda_{\mathrm{EL}}{ }^{\mathrm{LED}}=465\right.$ $\mathrm{nm}, \lambda_{\mathrm{abs}}{ }^{\text {DBrPTE }}=468 \mathrm{~nm}$ and $\lambda_{\mathrm{abs}}{ }^{\text {DBrPDI }}=528 \mathrm{~nm}$ ) for efficient excitation with the electroluminescence of blue LED (Figure 4a), the $\Phi_{\mathrm{PL}}$ of it is almost $20 \%$ of DBrPDI (Table).

\section{White light properties}

Drop casting the DBrPDI directly on blue LED produced white light with CIE coordinates of $\mathrm{x}=0.34, \mathrm{y}=0.29$, luminous and power efficiency values of $6.35 \mathrm{~cd} / \mathrm{A}$ and $2.22 \mathrm{~lm} / \mathrm{W} @ 5 \mathrm{~mA}$, respectively and CRI value of 60. However, drop casting DBrPTE on blue LED produced only yellow-green light with a peak maximum of $550 \mathrm{~nm}$, CIE coordinates of $\mathrm{x}=0.41 \mathrm{y}=0.57$ and efficiency values of $0.81 \mathrm{~lm} / \mathrm{W}$ and $2.20 \mathrm{~cd} / \mathrm{A} @ 5 \mathrm{~mA}$ (Figure 5). When compared with the bare DBrPTE PL wavelength range of $450 \mathrm{~nm}$ to $650 \mathrm{~nm}$ (Figure 4b), approximately $50 \mathrm{~nm}$ of red shift is obtained in InGaN/DBrPTE (Figure 5). This implies an aggregation induced emission $[15,23,24]$ and shows that 1,7-dibromination of perylene-3,4,9,10tetracarboxytetrabutylester could not interrupt $\pi-\pi$ stacking between the DBrPTE rings. Additionally, obtaining only yellow-green light suggests an efficient energy transfer resulting from the overlap between the EL spectrum of the LED and absorption of DBrPTE (Figure 4a) and that the entire EL energy is used in the excitation of ground state electrons of DBrPTE. Therefore, only DBrPDI derivative is mixed with the PMMA resin and applied on the blue LED. Although the current density (J)-voltage (V) characteristics of InGaN/DBrPDI and InGaN/DBrPDI:resin were almost identical (Figure 6a), InGaN/DBrPDI:resin sample presented much better white light efficiency values (Figure 6b-d). InGaN/DBrPDI:resin presented efficiency values of $23 \mathrm{~cd} / \mathrm{A}$ and $8.5 \mathrm{~lm} / \mathrm{W} @ 5 \mathrm{~mA}$ whereas these values were 6.35 
cd/A and 2.22 1m/W@5mA for the InGaN/DBrPDI sample. The resulted 3 orders of magnitude increase is attributed to the fabulous optical properties of PMMA and its ability to provide an inert matrix for homogenous distribution of perylene derivative in the matrix $[25,26]$. A maximum brightness value of $33450 \mathrm{~cd} / \mathrm{m}^{2}$ is obtained (Figure 6b). EL vs wavelength spectrum is provided in Figure 6e. EL peak maximum of $465 \mathrm{~nm}$ for the blue LED is observed and the PL wavelength range of DBrPDI is preserved (Figure $4 \mathrm{~b}$ vs Figure $6 e)$. CIE coordinates of blue LED deviated with the applied current whereas a negligible shift $(\Delta x, y=0.02,0.01)$ is observed from InGaN/DBrPDI:resin (Figure 6f). Increasing the applied current resulted in an increase in CRI from 60@5mA to 90@20mA. White light with CIE coordinates of $\mathrm{x}=0.34, \mathrm{y}=0.29, \mathrm{CRI}$ value of 90 and temperature of $4700 \mathrm{~K}$ could be obtained with the applied current of $20 \mathrm{~mA}$.

Above discussed results show that by simply bromination of the perylene diimide ring from 1 , 6 and/or 7 positions, performance values comparable with the perylene derivatives presented for down conversion in literature, which need at least one more synthetic step [9,11-13,27] and commercially available BASF Lumogen F Yellow 083 [13] can be obtained.

\section{Conclusion}

In this study two perylene derivatives DBrPDI and DBrPTE were synthesized and used as wavelength converters on a GaN/InGaN based blue LED. While the use of DBrPTE, presented only the PL characteristic of DBrPTE itself, using DBrPDI enabled obtaining white light. CRI values and CIE coordinates of the InGaN/DBrPDI:resin sample were comparable with its commercial analogs. Color temperature of $4700 \mathrm{~K}$ points out that it may mimic daylight and can be utilized in security lighting, display areas, etc. The 1, 6 (7) functionalized perylene derivatives used in this study hold the advantage of relatively easy modification to gain longer wavelength emitting perylene derivatives, those of which may allow obtaining color temperatures between $3100-4500 \mathrm{~K}$ and even $2000-3000 \mathrm{~K}$.

\section{References}

$1 \quad$ S Nakamura, Y Harada, M Seno, Appl. Phys. Lett. 58(18): 2021(1991).

2 S Nakamura, In Situ Monitoring of GaN Growth Using Interference Effects. Jpn. J. Appl. Phys. 30(7): 1348 (1991).

3 Yen WM, Shionoya S, Yamamoto H, Phosphor HandBook. Editor-in-Chief: MJ Weber, Taylor \& Francis Group CRC Press, Boca Raton, 2007, Chapter 1.

$4 \quad$ HS Jang, YH Won, DY Jeon, Appl. Phys. B Lasers Opt. 95(4): 715 (2009).

$5 \quad$ C-H Huang, W-R Liu, T-S Chan, Y-T Lai, Dalton Trans. 43: 7917 (2014).

6 C-Y Wang, T Takeda, OM ten Kate, R-J Xie, K Takahashi, N Hirosaki, J. Mater. Chem. C. 4(43): 10358 (2016). 
7 Y Liu, M Pan, QY Yang, L Fu, K Li, SC Wei, CY Su, Chem. Mater. 24(10): 1954 (2012).

8 D Van der Heggen, JJ Joos, DC Rodríguez Burbano, JA Capobianco, PF Smet, Materials (Basel). 10(8): 1 (2017).

9 F Galeotti, W Mróz, M Catellani, B Kutrzeba-Kotowska, E Kozma, J. Mater. Chem. C. 4(23): 5407 (2016).

10 RJ Cotter. 2003. US00657693(B2): 1.

11 M Mosca, F Caruso, L Zambito, B Seminara, R Macaluso, C Calì, E Feltin, Proc. SPIE - Int. Soc. Opt. Eng. 8767: 87670L (2013).

12 F Caruso, M Mosca, S Rinella, R Macaluso, C Calì, F Saiano, E Feltin, J. Electron. Mater. 45(1): 682 (2015).

13 F Caruso, M Mosca, R Macaluso, E Feltin, C Cali, Electron. Lett. 48(22): 1417 (2012).

14 M Mosca, F Caruso, L Zambito, R Macaluso, C Cal1, Photonics Spectra, 47(4), 60 (2013).

15 M Kus, Ö Hakli, C Zafer, C Varlikli, S Demic, S Özçelik, S Icli, Org. Electron. 9(5): 757 (2008).

16 C Karapire, M Kus, G Turkmen, CC Trevithick-Sutton, CS Foote, S Icli, Sol. Energy. 78(1): 5 (2005).

17 C Karapire, C Zafer, S İçli, S Içli, Synth. Met. 145(1): 51 (2004).

18 C Karapire, C Timur, S İçli, Dye. Pigment. 56(2): 135 (2003).

19 SA El-daly, TA Fayed, J. Photochem. Photobiol. A. 137: 15 (2000).

20 P Rajasingh, R Cohen, E Shirman, LJW Shimon, B Rybtchinski, J. Org. Chem. 72(16): 5973 (2007).

21 S Sengupta, RK Dubey, RWM Hoek, SPP van Eeden, DD Gunbas, FC Grozema, EJR Sudhölter, WF Jager, J. Org. Chem. 79: 6655 (2014).

22 H Langhals, J Karolin, LB-Å Johansson, J. Chem. Soc., Faraday Trans. 94(19): 2919 (1998).

23 PAJ De Witte, J Hernando, EE Neuteboom, EMH P van Dijk, SCJ Meskers, RAJ Janssen, NF van Hulst, RJM Nolte, MF García-Parajó, AE Rowan, J. Phys. Chem. B. 110(15): 7803 (2006).

24 C Burgdorff, HG Löhmannsröben, R Reisfeld, Chem. Phys. Lett. 197(4-5): 358 (1992).

25 AF Mansour, MG El-Shaarawy, SM El-Bashir, MK El-Mansy, M Hammam, Polym. Int. 51(5): 393 (2002).

26 WJ Wadsworth, SM Giffin, IT McKinnie, JC Sharpe, AD Woolhouse, TG Haskell, GJ Smith, Appl. Opt. 38(12): 2504 (1999).

27 E Kozma, W Mróz, F Galeotti, Dye. Pigment. 114: 138 (2015). 


\section{Figure Captions}

Figure 1. FTIR spectra of PTE, DBrPTE and DBrPDI

Figure 2. ${ }^{1} \mathrm{H}$ NMR spectrum of regioisomerically pure 1,7-dibromoperylene-3,4,9,10tetracarboxytetrabutylester (DBrPTE)

Figure 3. ${ }^{1} \mathrm{H}$ NMR spectrum of $\mathrm{N}, \mathrm{N}^{\prime}$-Bis(2,6-diisopropylphenyl)-dibromoperylene-3,4,9,10tetracarboxybisimide (DBrPDI)

Figure 4. a) Normalized EL and absorbance and b) Normalized EL and PL spectra of blue LED and synthesized perylene derivatives, respectively.

Figure 5. EL intensity spectra of InGaN/DBrPTE

Figure 6. a) Current density - potential, b) brightness- potential, c) power efficiencypotential, d) luminous efficiency- potential curves of InGaN, InGaN/DBrPDI and InGaN/DBrPDI:resin samples, e) EL intensity-Wavelength curve of InGaN/DBrPDI:resin sample and f) CIE coordinate deviations of blue LED and InGaN/DBrPDI:resin between the applied currents of $5 \mathrm{~mA}$ and $37 \mathrm{~mA}$. 


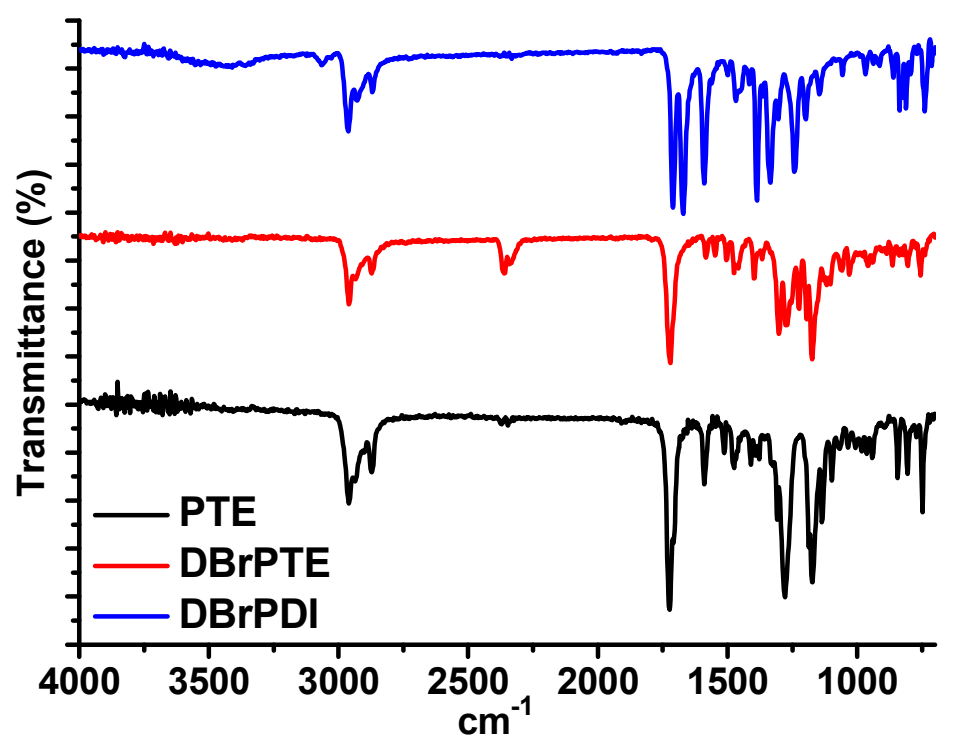

Figure 1. 


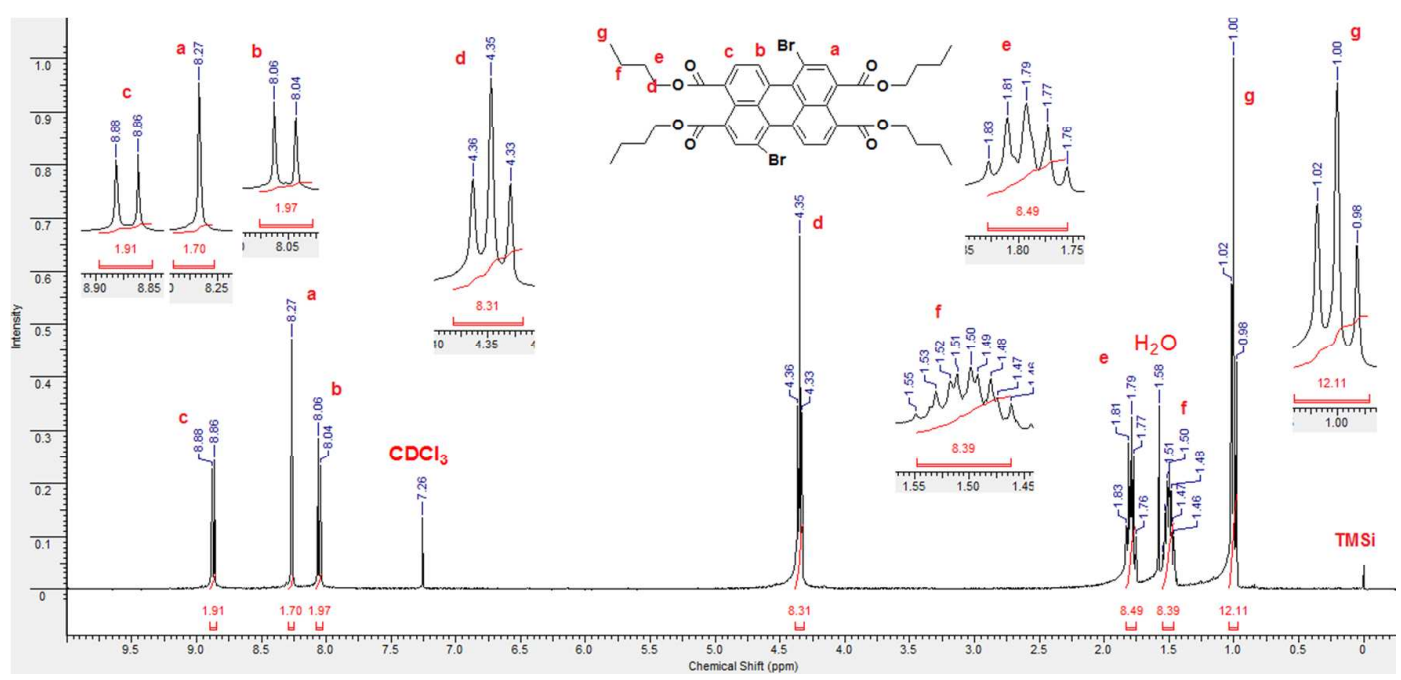

Figure 2. 


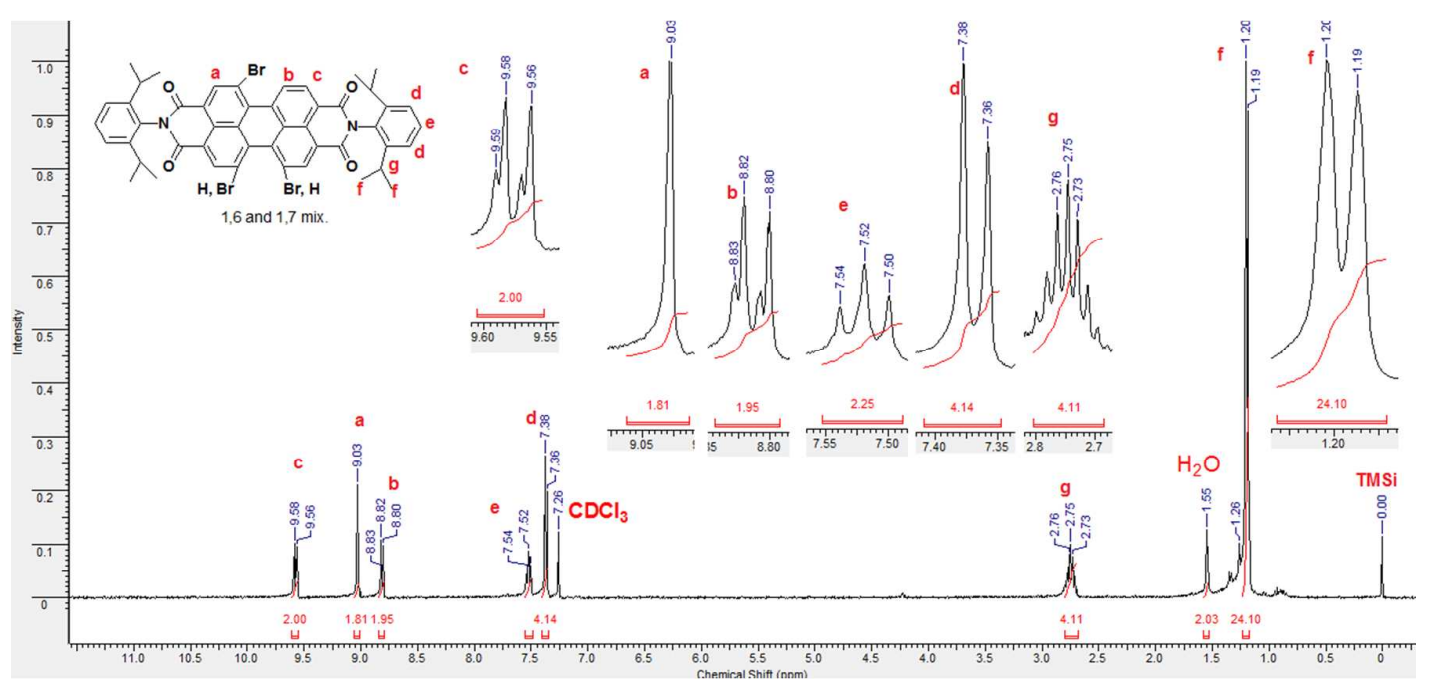

Figure 3. 

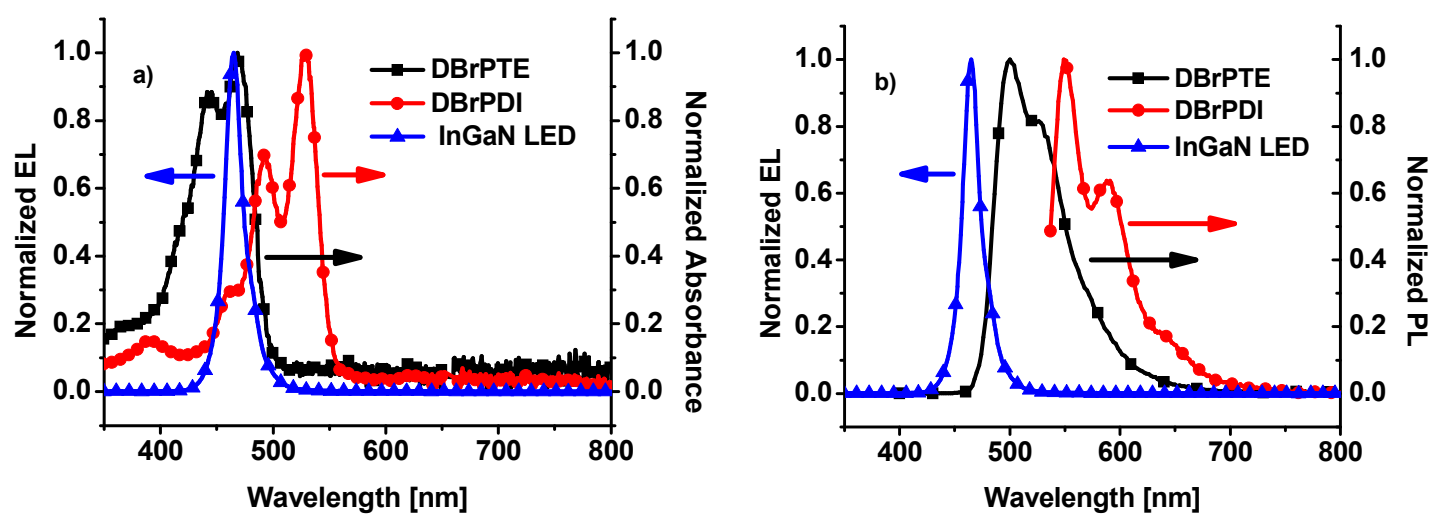

Figure 4. 


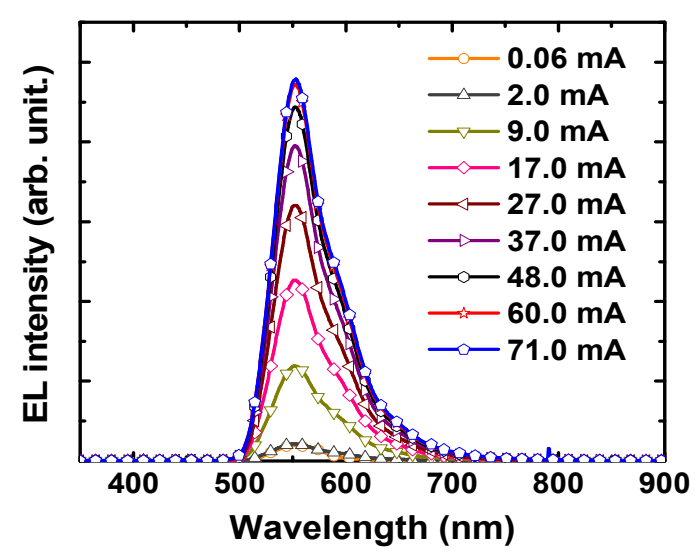

Figure 5. 

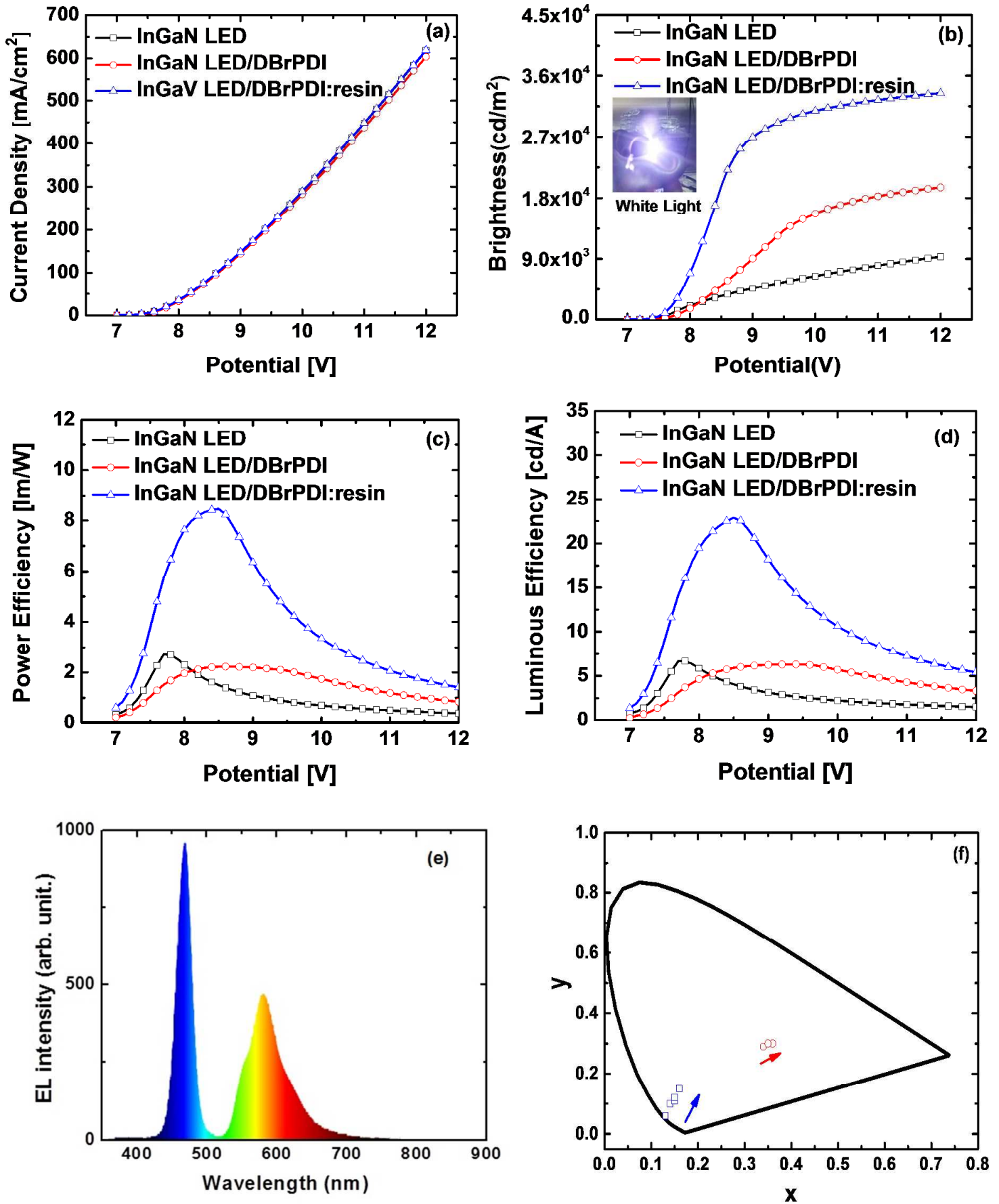

Figure 6. 
Table. Absorption wavelengths $\left(\lambda_{\text {abs }}, \mathrm{nm}\right)$, molar absorptivity constants $\left(\varepsilon, \mathrm{M}^{-1} \mathrm{~cm}^{-1}\right)$, singlet energies $\left(E_{\mathrm{s}}, \mathrm{kcal} / \mathrm{mol}\right)$, excitation wavelengths $\left(\lambda_{\text {exc }}, \mathrm{nm}\right)$, photoluminescence quantum yields $\left(\Phi_{\mathrm{PL}}\right)$ and fluorescence life times $\left(\tau_{\mathrm{f}, \mathrm{ns}}\right)$ of perylene derivatives.

\begin{tabular}{|c|c|c|c|c|c|c|c|c|c|}
\hline $\begin{array}{c}\text { Perylene } \\
\text { derivatives }\end{array}$ & $\begin{array}{l}\lambda_{\text {abs }} \\
(\mathrm{nm})\end{array}$ & $\begin{array}{c}\operatorname{Ex} 10^{-4} \\
\left(\mathrm{M}^{-1} \mathrm{~cm}^{-1}\right)\end{array}$ & $\begin{array}{c}\mathrm{E}_{\mathrm{s}}^{\mathrm{a}} \\
\text { (kcal/mol) }\end{array}$ & $\begin{array}{l}\lambda_{\text {exc }} \\
(\mathrm{nm})\end{array}$ & $\begin{array}{l}\lambda_{\text {ems }} \\
(\mathrm{nm})\end{array}$ & \multicolumn{2}{|c|}{$\Phi_{\mathrm{PL}}$} & \multicolumn{2}{|c|}{$\begin{array}{c}\tau_{\mathrm{f}} \\
(\mathrm{ns})\end{array}$} \\
\hline \multirow{2}{*}{ DBrPTE } & 445 & 2.40 & \multirow{2}{*}{60.90} & \multirow{2}{*}{468} & 500 & \multirow{2}{*}{0.11} & \multirow{2}{*}{$0.26^{b}$} & \multirow{2}{*}{$1.63^{\mathrm{c}}$} & \multirow{2}{*}{$1.78^{b}$} \\
\hline & 468 & 2.75 & & & 526 & & & & \\
\hline \multirow{3}{*}{ DBrPDI } & 462 & 1.21 & \multirow{3}{*}{54.89} & \multirow{3}{*}{528} & 550 & \multirow{3}{*}{0.50} & \multirow{3}{*}{$0.79^{b}$} & \multirow{3}{*}{$5.8^{\mathbf{c}}$} & \multirow{3}{*}{$4.36^{\mathrm{b}}$} \\
\hline & 492 & 3.18 & & & 589 & & & & \\
\hline & 528 & 5.04 & & & 642 & & & & \\
\hline
\end{tabular}

${ }^{a}$ calculated from the onset wavelength of photoluminescence curves

${ }^{\mathrm{b}}$ ref [21] (relative $\Phi_{\mathrm{PL}}$ determined by the comparative method using perylene-3,4,9,10tetracarboxylictetramethyl ester $\left(\Phi_{\mathrm{f}}=0.95\right.$ in $\left.\mathrm{CH}_{2} \mathrm{Cl}_{2}\right)$ as a reference [22])

${ }^{\mathrm{c}} 472.4 \mathrm{~nm}$ excitation laser is used 